\title{
Computer-Assisted Propofol Administration
}

\author{
J.P.A. O'Connor ${ }^{\mathrm{a}}$ C.A. O'Moráin ${ }^{\mathrm{a}}$ J.J. Vargo ${ }^{\mathrm{b}}$ \\ ${ }^{a}$ Department of Gastroenterology, Adelaide and Meath Hospital, Trinity College Dublin, Dublin, Ireland; \\ ${ }^{b}$ Department of Gastroenterology and Hepatology, Cleveland Clinic Lerner College of Medicine of \\ Case Western University, Cleveland, Ohio, USA
}

\section{Key Words}

Bispectral index $\cdot$ Computer-based platforms $\cdot$ Closed-loop target-controlled system

\begin{abstract}
The use of propofol for sedation in endoscopy may allow for better quality of sedation, quicker recovery and facilitate greater throughput in endoscopy units. The cost-effectiveness and utility of propofol sedation for endoscopic procedures is contingent on the personnel and resources required to carry out the procedure. Computer-based platforms are based on the patients response to stimulation and physiologic parameters. They offer an appealing means of delivering safe and effective doses of propofol. One such means is the bispectral index where continuous EEG recordings are used to assess the degree of sedation. Another is the closedloop target-controlled system where a set of physical parameters, such as muscle relaxation and auditory-evoked potential, determine a level of medication appropriate to achieve sedation. Patient-controlled platforms may also be used. These electronic adjuncts may help endoscopists who wish to adopt propofol sedation to change current practices with greater confidence.

Copyright $\odot 2010$ S. Karger AG, Basel
\end{abstract}

\section{Introduction}

The current standard of care for endoscopic sedation is conscious sedation, usually with a benzodiazepine and/ or opioid analgesia. Propofol has been proposed as a suitable alternative agent for sedation in endoscopy, which may allow for better quality of sedation, quicker recovery and facilitate greater throughput in endoscopy units. Its advantages are that it has rapid onset (1-2 min), brief duration (5-10 $\mathrm{min}$ ) and fast recovery (15-20 $\mathrm{min})$. It rapidly crosses the blood-brain barrier and causes a depression in consciousness that is likely related to potentiation of the $\gamma$-aminobutyric acid A receptor in the brain [1]. During endoscopist-directed propofol sedation, it is typically administered in small bolus doses either alone or with the use of a short-acting opiate and/or benzodiazepine.

Propofol is estimated to be the primary agent used in $25 \%$ of endoscopies in the United States [2]. The efficacy of propofol as a sedative agent for endoscopy has become well-established in recent years. A study of 75 patients receiving advanced upper endoscopy in 2002 showed similar physiological outcomes and satisfaction in patients receiving propofol from gastroenterologists compared to those receiving benzodiazepines. However, patients receiving propofol had shorter recovery times and

Anthony O'Connor

Department of Gastroenterology

Adelaide and Meath Hospital, Trinity College Dublin

Belgard Road, Tallaght, Dublin 24 (Ireland)

Tel. +353 1414 2000, Fax +35314143850, E-Mail oconna12@tcd.ie 
a higher recovery of both baseline activity level and dietary intake $24 \mathrm{~h}$ after the procedure [3].

The cost-effectiveness and utility of propofol sedation in patients undergoing endoscopic procedures is contingent on the personnel who can carry out the procedure. Propofol is administered by the gastroenterologist or a trained endoscopy nurse in the majority of patients, with more complex cases often being administered by an anaesthesiologist.

\section{Computer-Based Platforms for Sedation}

Computer-based platforms offer a very appealing means of delivering safe doses of propofol in a cost-effective manner. These are based on the patients response to auditory and tactile stimulation as well as physiologic parameters [4]. In a joint study of 96 patients undergoing simple diagnostic endoscopy in the USA and Belgium, the total dose and range of propofol dosing in the US and Belgian studies was 70.4 and $76.7 \mathrm{mg}$, respectively. There were a total of 8 episodes of transient oxygen desaturation and 73 episodes of transient apnoea lasting 1-37 s. Patients remained moderately sedated throughout the examination. Recovery time, defined by an Aldrete score of 12 or higher, was less than $1 \mathrm{~min}$ for the majority of patients. There were no serious drug- or device-related events, and intervention by an anaesthesiologist was not required [5].

\section{Bispectral Index}

Another computer-based intervention is the bispectral index. In this instance, continuous EEG recordings are used to generate an objective assessment of the degree of sedation. The computer signals the infusion pump to deliver the amount of drug necessary to achieve the predetermined drug concentration. Adjustments can be made for weight, co-morbidity, gender and other pharmacotherapeutic factors.

Bispectral index platforms have been shown to correlate well with subjective measurements of sedation such as the Observer's Assessment of Alertness/Sedation (OAA/S) Scale [6]. To adjust for individual variation, the patient must be monitored continuously and adjustments made as necessary to the target concentration [7]. Some studies have suggested a significant lag time compared with the clinical assessment of the level of sedation. The use of the bispectral index also did not lead to reductions in mean propofol dose or recovery time when used as an adjunct to nurse-administered propofol sedation for colonoscopy, or when used as the primary target for sedation $[8,9]$.

\section{Closed-Loop Target-Controlled System}

A variation on this theme is the concept of a closedloop target-controlled system. In this case, the operator selects a set of physical parameters, such as muscle relaxation and auditory-evoked potential, which are continuously monitored. The system integrates this information and selects a level of medication appropriate to achieve the desired level of sedation. In theory this should reduce the potential for under- and over-sedation [6]. One study assessed 205 patients undergoing ERCP using TCI (target-controlled infusion) of propofol. An initial plasma concentration of $4 \mathrm{~g} / \mathrm{ml}$ was targeted and the dose was subsequently titrated between 2 and $5 \mathrm{~g} / \mathrm{ml}$ by an anaesthesiologist to maintain patient cooperation and comfort. A single bolus of fentanyl (50-100 g) was provided if patients showed signs of inadequate analgesia. The quality of sedation was considered excellent in 201 of 205 patients by both the endoscopist and the nurse [10].

In a comparative study, TCI of propofol was compared with anaesthesiologist-administered propofol in a study of 40 patients undergoing colonoscopy. The initial target plasma concentration of propofol was set at $0.8 \mathrm{~g} / \mathrm{ml}$, with increments of $0.1 \mathrm{~g} / \mathrm{ml}$ triggered by a patient-controlled handset. Patients in the TCI group were sedated more slowly and less deeply, and received a lower total dose of propofol than the anaesthesiologist-administered propofol group. Patient satisfaction and recovery times were comparable in the two groups [11]. The closed-loop system has also been tested. In one small study, 16 patients received TCI of propofol using a closed-loop system guided by the bispectral index. In this admittedly small group, no patient became haemodynamically unstable or required airway support, and patient and physician satisfaction were high [12].

\section{Patient-Controlled Sedation}

Patient-controlled sedation is another means by which a computerised device can assist in the administration of propofol. One study using propofol/alfentanil in 16 patients undergoing colonoscopy showed a recovery time of $9 \mathrm{~min}$, patient satisfaction with the degree of sedation and 
comfort achieved, and no significant adverse events [13]. In a second study, the same investigators compared patient-controlled administration with conventional sedation using pethidine and a benzodiazepine in 66 patients undergoing colonoscopy. The patients receiving patientcontrolled analgesia/sedation were sedated more lightly and experienced slightly more pain during the examination, but recovered more quickly. Patients in both treatment arms were satisfied with the sedation [14].

\section{Conclusions}

The future of endoscopic sedation involves agents which are fast-acting, allow prompt recovery and give sedation of sufficient depth to ensure comfort, but are as a priority at least as safe as current methods. Of all the drugs in the current arsenal available to endoscopists, propofol is the best fit. The main limiting factor against its use at present is a lack of familiarity with the medication amongst most endoscopists and a perceived greater risk of adverse events - an opinion which has not been borne out by any of the evidence obtained from controlled clinical trials.

The use of computer-based platforms offers the endoscopist a useful adjunct in sedation and the use of this technology may inspire confidence in the practice of sedation with what is currently an unfamiliar agent to the majority of gastroenterologists. Further studies are needed on the safety and cost-effectiveness of computer-based platforms in endoscopic sedation.

\section{References}

$>1$ Trapani G, Altomare C, Liso G, et al: Propofol in anesthesia: mechanism of action, structure-activity relationships, and drug delivery. Curr Med Chem 2007;7:249-271.

$\checkmark 2$ Cohen LB, Wecsler JS, Gaetano JN, Benson AA, Miller KM, Durkalski V, Aisenberg J: Endoscopic sedation in the United States: results from a nationwide survey. Am J Gastroenterol 2006;101:967-974.

3 Vargo JJ, Zuccaro G Jr, Dumot JA, Shermock KM, Morrow JB, Conwell DL, Trolli PA, Maurer WG: Gastroenterologist-administered propofol versus meperidine and midazolam for advanced upper endoscopy: a prospective, randomized trial. Gastroenterology 2002;123:8-16.

$\checkmark 4$ Doufas AG, Bakshandeh M, Bjorsten AR, Sahfer DI: Induction speed is not a determinant of propofol pharmacodynamics. Anesthesiology 2004;101:1112-1121.

5 Pambianco DJ, McRorie J, Martin J, Politove R, Whitten CJ: Feasibility assessment of computer-assisted personalized sedation: a sedation delivery system to administer propofol for gastrointestinal endoscopy (abstract). Gastrointest Endosc 2006;63:AB189.
6 Bower AL, Ripepi A, Dilger J, Boparai N, Brody FJ, Ponsky JL: Bispectral index monitoring of sedation during endoscopy. Gastrointest Endosc 2000;52:192-196.

7 Egan TD: Target-controlled drug delivery: progress toward an intravenous 'vaporizer' and automated anesthetic administration. Anesthesiology 2003;99:1214-1219.

8 Drake LM, Chen SC, Rex DK: Efficacy of bispectral monitoring as an adjunct to nurseadministered propofol sedation for colonoscopy: a randomized controlled trial. Am J Gastroenterol 2006;101:2003-2007.

$>9$ Chen SC, Rex DK: An initial investigation of bispectral monitoring as an adjunct to nurseadministered propofol sedation for colonoscopy. Am J Gastroenterol 2004;99:10811086.

10 Fanti L, Agostoni M, Casati A, Guslandi M, Giollo P, Torri G, Testoni PA: Target-controlled propofol infusion during monitored anesthesia in patients undergoing ERCP. Gastrointest Endosc 2004;60:361-366.
1 Stonell CA, Leslie K, Absalom AR: Effect-site targeted patient controlled sedation with propofol: comparison with anaesthetist administration for colonoscopy. Anaesthesia 2006;61:240-247.

12 Leslie K, Absalom A, Kenny GN: Closed loop control of sedation for colonoscopy using the Bispectral Index. Anaesthesia 2002;57:693697.

13 Roseveare C, Seavell C, Patel P, Criswell J, Kimble J, Jones C, et al: Patient-controlled sedation and analgesia, using propofol and alfentanil, during colonoscopy: a prospective randomized controlled trial. Endoscopy 1998;30:768-773.

14 Bright E, Roseveare C, Dalgleish D, Kimble J, Elliott J, Shepherd H: Patient-controlled sedation for colonoscopy: a randomized trial comparing patient-controlled administration of propofol and alfentanil with physician-administered midazolam and pethidine. Endoscopy 2003;35:683687. 